\title{
Computer-Assisted Surgery for Mandibular Reconstruction Using a Patient-Specific Titanium Mesh Tray and Particulate Cancellous Bone and Marrow
}

\author{
Seiji Kondo ${ }^{1}$, Hideyuki Katsuta ${ }^{2}$, Ayako Akizuki' ${ }^{1}$, Yuji Kurihara1, Takaaki Kamatani ${ }^{1}$, \\ Atsushi Yaso², Masahiro Nagasaki1, Toshikazu Shimane' ${ }^{2}$, Tatsuo Shirota1 \\ ${ }^{1}$ Division of Craniomaxillofacial Surgery, Department of Oral and Maxillofacial Surgery, School of Dentistry, \\ Showa University, Tokyo, Japan \\ ${ }^{2}$ Division of Oral Oncology, Department of Oral and Maxillofacial Surgery, School of Dentistry, Showa University, \\ Tokyo, Japan \\ Email: seikondo12@dent.showa-u.ac.jp
}

Received 20 February 2015; accepted 6 March 2015; published 10 March 2015

Copyright $@ 2015$ by authors and Scientific Research Publishing Inc.

This work is licensed under the Creative Commons Attribution International License (CC BY). http://creativecommons.org/licenses/by/4.0/

(c) (i) Open Access

\section{Abstract}

Craniomaxillofacial surgery is difficult due to the complexity of the regional anatomy. Computerassisted surgery is a promising tool aiming to improve the safety and precision of such surgery. A computer-assisted surgical navigation approach for reconstruction of mandibular defects using a patient-specific titanium mesh tray and particulate cancellous bone and marrow (PCBM) harvested from bilateral anterior ilia is proposed. This case report involves a large multicystic ameloblastoma affecting the right mandible of a 31-year-old male patient. Following detailed clinical examination, radiological interpretation, and histopathological diagnosis, computer-assisted surgical simulation with a virtual 3-dimensional (3-D) model was designed using surgical planning software based on the pre-operative computed tomography data. Long-span segmental resection of the mandible was planned, and the defect was analyzed for reconstruction using a patient-specific reconstruction titanium mesh tray mediated with computer-aided design and manufacturing (CAD/CAM) techniques. During the actual surgery, the ultrasonic bone cutting instrument in the surgeon's hand was connected to the navigation system to touch an anatomical position on the patient. Therefore, osteotomies were performed finely and smoothly according to the navigation images of the cutting bone line by sequentially moving the instrument. Finally, a CAD/CAM-mediated titanium mesh tray condensed by PCBM was adapted to the remaining mandibular fragments. Six months postoperatively, the patient had a good mandibular configuration and facial contour. Integration

${ }^{*}$ Corresponding author.

How to cite this paper: Kondo, S., et al. (2015) Computer-Assisted Surgery for Mandibular Reconstruction Using a PatientSpecific Titanium Mesh Tray and Particulate Cancellous Bone and Marrow. Case Reports in Clinical Medicine, 4, 85-92. 
of different technologies, such as software planning and 3-D surgical simulation, combined with intraoperative navigation and CAD/CAM techniques, provides safe and precise mandibular reconstruction surgery.

\section{Keywords}

\section{Patient-Specific Titanium Mesh Tray, Computer-Assisted Surgery, Mandibular Reconstruction, Particulate Cancellous Bone and Marrow, Surgical Navigation}

\section{Introduction}

Craniomaxillofacial surgery is difficult due to the dangerous structures in the compact and deeply hidden region [1]. In the past, surgery in this area was performed by conventional methods, so-called "free hand", depending on surgeons' competence, experience, and 2-dimensional (2-D) imaging. However, in recent years, advanced engineering technology including medical image processing, computer-aided design and computer-aided manufacturing $(\mathrm{CAD} / \mathrm{CAM})$, rapid prototyping $(\mathrm{RP})$ known as 3-D printing, and surgical navigation have been widely used in the fields of neuro- [2], orthopedic- [3], and otolaryngological surgery [4]. Of these advanced techniques, virtual surgical simulation before the actual surgery progressing from 2-D images to sophisticated 3-D images and surgical navigation providing real-time feedback to the surgeons dramatically improve the quality and efficiency of the actual surgery. However, these computer-assisted surgery techniques are still in their preliminary stages in the field of craniomaxillofacial surgery [5].

The mandible plays an important role in facial symmetry, occlusion, mastication, and speech functions [6]-[9]. Defects of the mandible caused by tumor, osteomyelitis, or severe trauma affect patients' quality of life both physiologically and psychologically. Various approaches for mandibular reconstruction, including reconstructive titanium plates, nonvascularized or vascularized bone grafts, and autogenous iliac bone grafts in combination with a ready-made titanium mesh trays, have been tried [10] [11]. The most common approach for mandibular reconstruction today is a vascularized-bone graft from the fibula and iliac crest [6]-[8]. However, the area for resection of the mandible cannot be positioned precisely even with any of the approaches described above, and the complexity of the regional anatomy makes it very difficult to reconstruct the facial contour and recover ideal occlusal function. For example, due to the straight configuration of the fibula, surgical time and the risk of vascular complications are increased. To completely resolve these problems, advanced engineering technology has been used for mandibular resection and reconstruction in the clinic. Preoperative computer design, including prebending reconstruction materials combined with intraoperative navigation, provides a more accurate, safer, and less time-consuming method for reconstruction of the mandible compared with conventional methods of flap insertion.

In the present report, computer-assisted mandibular resection with the guidance of real-time navigation and reconstruction using a patient-specific titanium mesh tray and particulate cancellous bone and marrow (PCBM) harvested from bilateral anterior ilia is reported. The combination of surgical simulation with a virtual 3-D model, intraoperative navigation with real-time feedback to the surgeons, and CAD/CAM technique provides a safe and precise method for mandibular reconstruction.

\section{Case Report}

\subsection{Patient, Diagnosis and Treatment Plan}

A 31-year-old man was referred to our department, with a chief complaint of swelling of the right mandible for 6 months. On extraoral examination, there was a diffuse bony swelling of the right mandible. Intraoral examination revealed bicortical expansion of the alveolar ridge of the right mandible, with the swelling extending from the third molar to the canine. A part of the tumor-like mass with absence of ulcerations was observed in the mucosa of the right buccal vestibule.

The patient's past medical history was unremarkable. A diagnosis of multicystic ameloblastoma was made based on the histological, radiological, and clinical findings. Long-span segmental resection of the mandible was planned 
to excise the tumor mass completely. To provide safe and precise surgery, resection of the mandible was performed using intraoperative navigation. Simultaneously, computer-assisted mandibular reconstruction using a custom-made titanium mesh tray and particulate cancellous bone and marrow (PCBM) harvested from bilateral anterior ilia was selected due to the advantages of pre-producing the natural configuration of the mandible, no need for special surgical techniques such as anastomosis, and no postoperative complications associated with a bone donor site.

\subsection{Technical Procedure of Mandibular Resection and Reconstruction}

The outline of the technical procedure comprised two steps: virtual simulation and fabrication (Figure 1). First, the patient was scanned using a computed tomography (CT) scanner (HiSpeed QX/i, GE Healthcare, Milwaukee, WI, USA) with a $25-\mathrm{cm}$ field of view and dentition mode. Each slice was composed of $512 \times 512$ pixels, and the pixel size was $0.49 \mathrm{~mm} \times 0.49 \mathrm{~mm}$. Slice thickness was $1.25 \mathrm{~mm}$. To determine a stable and reproducible mandibular position, positioning the mandible in centric relation occlusion was determined using a dental splint before the CT scan [8] [12] [13]. In the virtual simulation step, the digital imaging and communications in medicine (DICOM) data were imported into the 3-D planning software (IPLAN ENT/CMF ${ }^{\circledR}$ ver. 3.0.3, BrainLab, Feldkirchen, Germany), and a virtual 3-D model of the mandible was generated by semiautomatic thresholding and volumetric surface rendering, and all aspects of the digital plan were approved (Figure 2(a)). On this 3-D image, the original model was separated into the defective and healthy parts of the mandible to design a virtual repaired model. Based on cutting paths at the margin of the defective mandible, the tumor defect area was removed, and the virtual repaired model was generated using the mirror image of the healthy side of the mandible. The virtual repaired model provided the basic information for the design of a patient-specific reconstruction tray. In the fabrication steps, the physical models of the original and repaired mandibular models were fabricated by a 3-D printer to optimize osteotomy placement and position of the remaining mandibular fragments. The physical model of the repaired mandible was used to generate a patient-specific reconstruction tray, an ultra flex mesh tray that was fabricated from 3-D formable titanium mesh using a forming-hand press machine and CAD/CAM

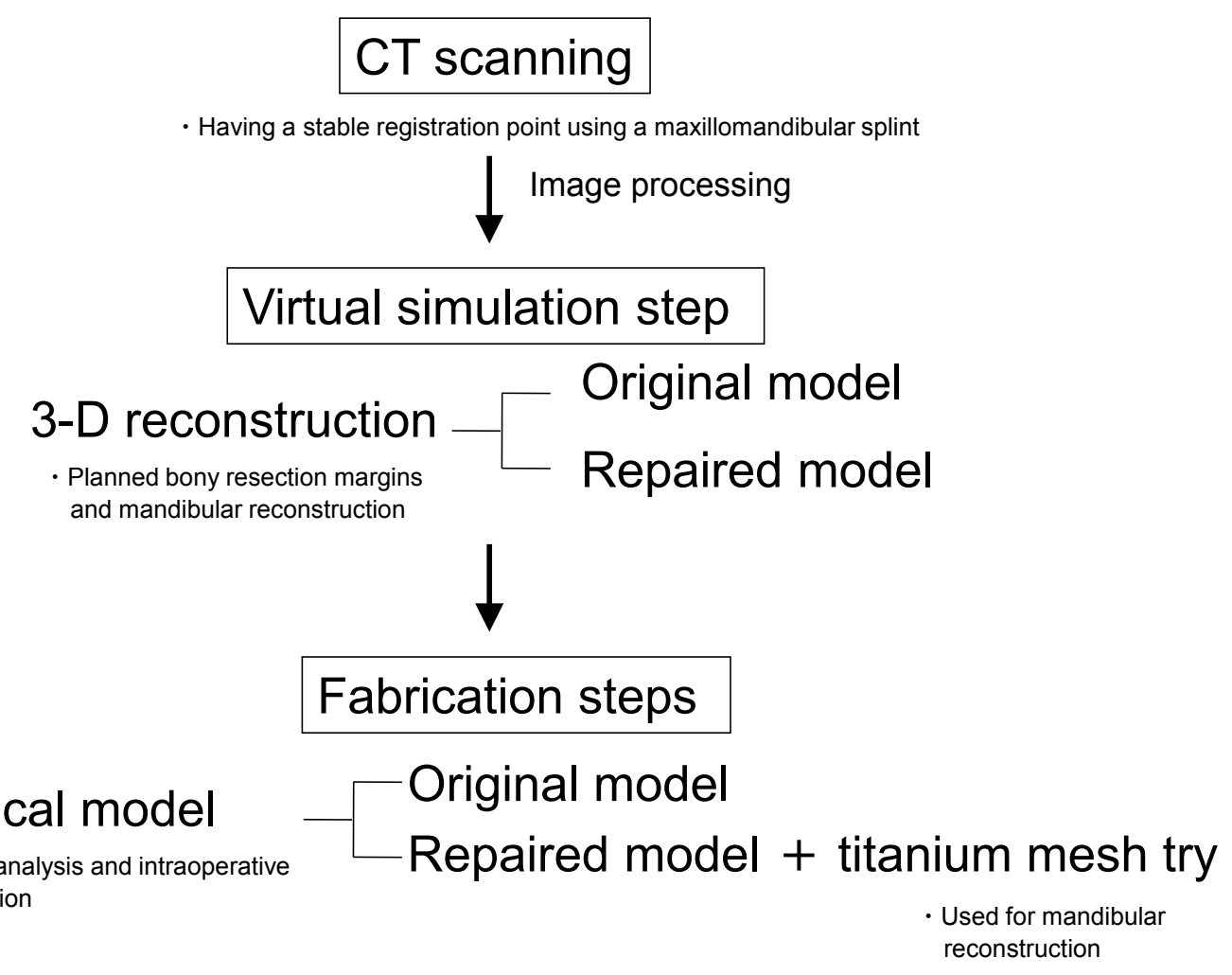

Figure 1. Outline of the computer-assisted presurgical procedure. Abbreviations: CT, computed tomography; 3 D, 3-dimensional. 


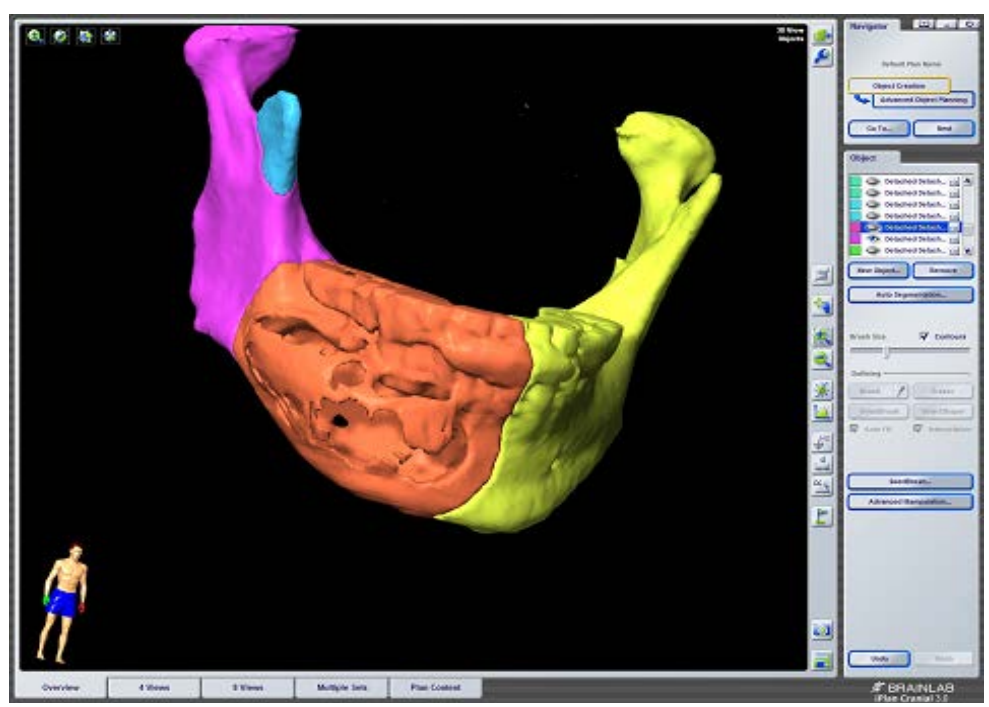

(a)

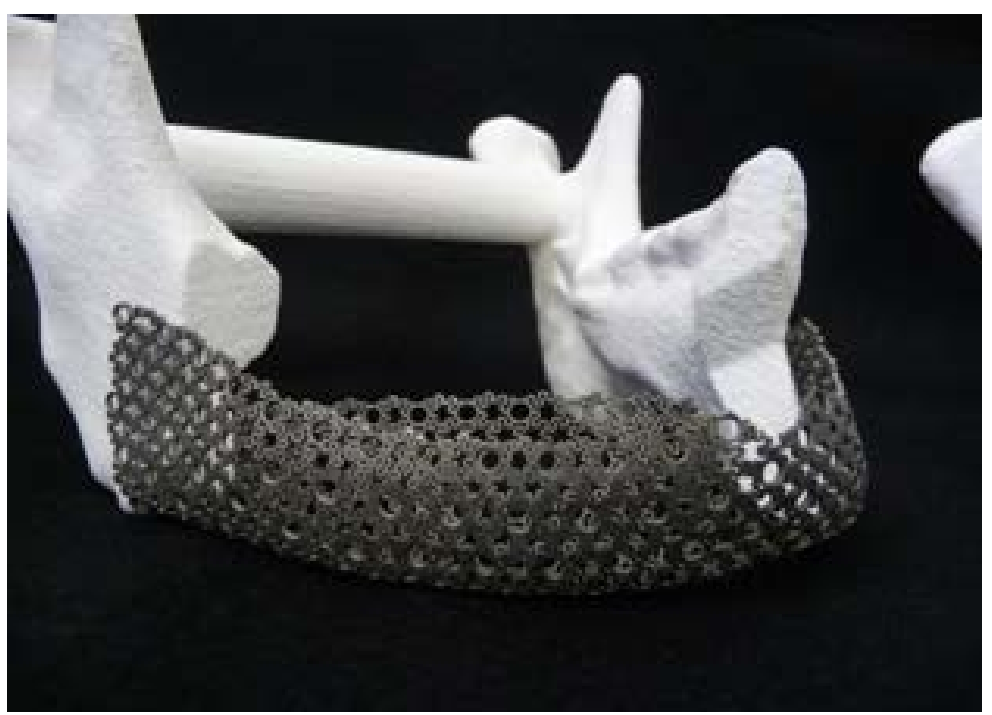

(b)

Figure 2. Computer-assisted presurgical planning. (a) Virtual resection of the right mandible. Computed tomographic images in DICOM format imported into IPLAN ENT/CMF ${ }^{\circledR}$ ver. 3.0.3. Software. The surgical resection is planned, and the expected defect (orange) and coronoid process (blue) treated with coronoidectomy are highlighted. (b) Design of the custom-made titanium mesh tray that was fabricated from 3-D formable titanium mesh (margaret flowers pattern) using a forming-hand press machine and computer-aided design and computer-aided manufacture (CAD/CAM). The titanium mesh tray fits the physical model of the repaired mandible.

techniques (Okada Medical Supply Co., Ltd., Tokyo, Japan) (Figure 2(b)). Conventional bending technique performed by a dental technician was not needed. Fabrication of a surgical template for bone cutting guides was also not needed due to the surgical navigation system.

\subsection{Intraoperative Navigation}

The surgical navigation system (Kick navigation system ${ }^{\circledR}$, BrainLab) including a tracking device was used to transfer the pre-operative design to the actual surgery, and passive signals were received simultaneously from the surgical instrument on the surgeon's hand. The pre-operative CT images were displayed on the monitor of IPLAN Screen Shots ${ }^{\circledR}$ (BrainLab). With the patient under general anesthesia, the reference arc was attached 
to the patient's forehead using a headband, and maxillomandibular fixation was performed using a dental splint with recorded centric relation occlusion to optically track mandible position. The navigation system could locate the head reference in the coordinate system of the CT data set through successive transform manipulation. Briefly, the tip of the Vector Vision II (BrainLab) pointer was placed on a specific anatomic landmark: the skin on the bilateral zygomatic arch regions, the upper central incisor and bilateral upper first moler, and the position of the pointer tip in the patient space was calculated automatically through the navigation system and transferred to the corresponding position in the preoperative CT image space. Subsequently, the ultrasonic bone cutting instrument, Sonopet ${ }^{\circledR}$ (Stryker, Kalamazoo, MI, USA), could be located and tracked in the coordinate system of the CT data, based on the position of the instrument relative to the head reference.

\subsection{Surgical Procedure}

A submandibular approach without identifying the facial nerve was used to access the affected mandibular body site, and segmental resection of the mandible was performed. The resection margin based on the pre-operative design was delineated according to the real-time intraoperative imaging by sequentially moving the Sonopet ${ }^{\circledR}$ on the surgeon's hand (Figure 3(a) and Figure 3(b)). After resecting the canine anteriorly in the left mandible, the third molar posteriorly in the right mandible was resected with no preservation of the neurovascular bundle (Figure 3(c)). Subsequently, preparation of the mandibular reconstruction was initiated. After condylar repositioning using a premade centric relation occlusion recording [12], a patient-specific titanium mesh tray was positioned to the residual mandibular fragments for a trial. PCBM was harvested from the lateral edges of bilateral anterior iliac crests. Finally, the titanium mesh tray condensed by PCBM was adapted to the remaining mandibular fragments and fixed using several monocortical screws.

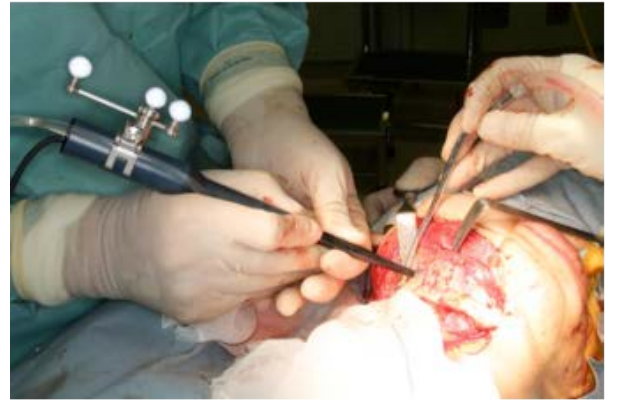

(a)

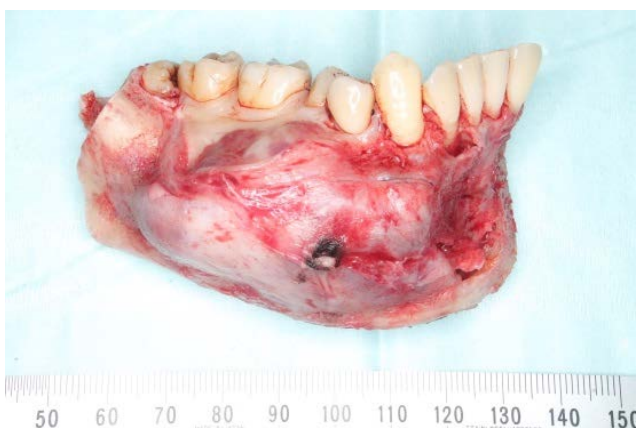

(c)

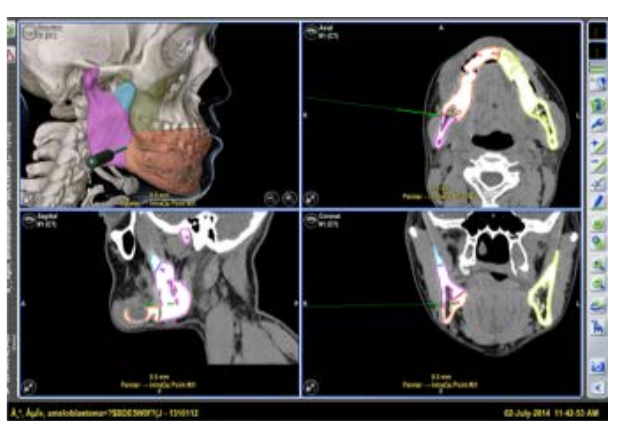

(b)

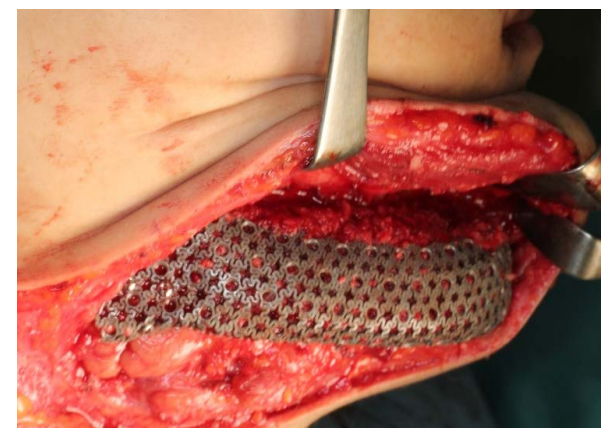

(d)

Figure 3. Intraoperative navigation for segmental resection of the mandible and reconstruction using a custommade titanium mesh tray and particulate cancellous bone and marrow. (a) Navigated surgical resection. Navigation tool attached to the Sonopet ${ }^{\circledR}$ on the surgeon's hand. Osteotomies were performed finely and smoothly according to the navigation images of the cutting bone line by sequentially moving the instrument. (b) Proper horizontal, sagittal, and vertical positioning of the cutting bone line is simultaneously confirmed using intraoperative navigation. The operative anatomy seen in (a) is accurately matched on the CT images on the navigation screen. (c) Resection specimen. Segmental resection of the mandible is performed precisely according to the navigation images of the cutting bone line. (d) Intraoperative view showing the custom-made titanium mesh tray. The PCBM is loaded and condensed into the tray. 


\subsection{Ethics}

Informed consent for including the patient's photographs was obtained from the patient.

\section{Clinical Results}

Operations during the clinical course of this case are shown in Figure 3. First, the right mandibular body was opened and exposed, and then the segmental resection of the defective mandible was performed safely and precisely with the guidance of real-time navigation. The resection curve was performed between the third molar posteriorly in the right mandible to the canine anteriorly in the left mandible for full excision of the tumor on a virtual 3-D model, and an accurate match between the operative anatomy and the CT images was achieved within $1.0 \mathrm{~mm}$. Furthermore, unlike rotational reciprocating motion, the cutting action of bone using the Sonopet $^{\circledR}$ was performed finely and sequentially with no grabbing or kicking.

Subsequently, reconstruction of the mandible was undertaken. PCBM was harvested from the lateral edges of bilateral anterior iliac crests. The amount of harvested PCBM was $40.0 \mathrm{~g}$. A patient-specific titanium mesh tray was accurately positioned to the remaining mandibular fragments without difficulty and fixed using several monocortical screws without positional change of the condyle (Figure 3(d)). The PCBM was loaded into the tray and densely condensed to increase graft density. The surgical simulation with the virtual 3-D model and surgical navigation provided safe and precise mandibular reconstruction. Six months postoperatively, both facial contour recovery (Figure 4(a) and Figure 4(b)) and occlusal rehabilitation (Figure 4(c) and Figure 4(d)) were achived, showing excellent new bone formation of the mandible radiologically (Figure 4(e)).

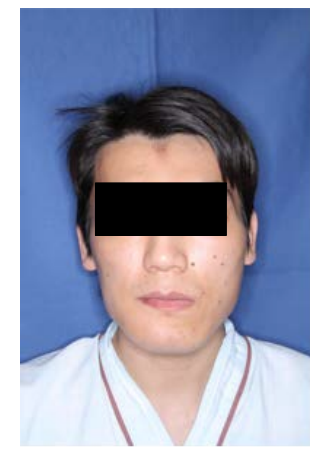

(a)

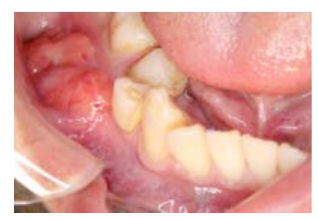

(c)

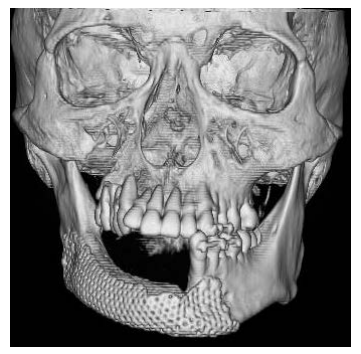

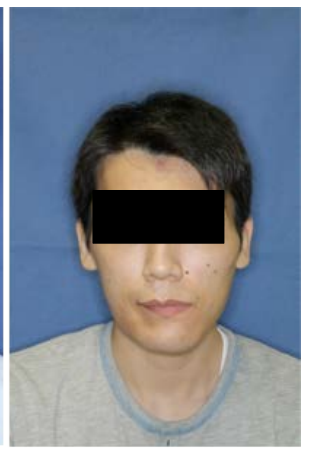

(b)

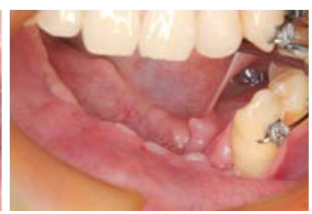

(d)

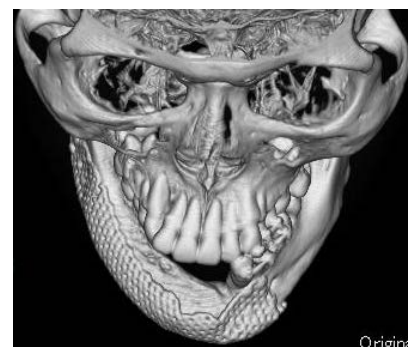

(e)

Figure 4. Comparison of preoperative and postoperative views. (a) Preoperative facial appearance. (b) Facial appearance at 6 months postoperatively. The patient's facial aesthetics have been restored. (c) Preoperative intraoral appearance. (d) Intraoral appearance at 6 months postoperatively. (e) Postoperative CT scanning with 3 dimension reconstruction showing excellent new bone formation. 


\section{Discussion}

This is the first report describing how computer planning and intraoperative navigation are used for reconstruction of the mandible with a patient-specific titanium mesh tray and PCBM. The proposed protocol incorporated all of the latest technologies and improved the safety and precision of the mandibular reconstruction surgery. It had been generally considered that a navigation system could not be used in mandibular surgery because of the complexities of navigating a mobile structure [5] [8]. However, these limitations were overcome to have a stable registration point using a maxillomandibular splint before CT was performed [8] [12]. This method did not take any additional cost and had no invasive action. Even if an open jaw surgery was required, the intraoperative navigation surgery was possible to undergo with a splint reproducing the same open-jaw position used during the CT scan. In fact, Ohba et al. [13] applied the navigation system to mandibular surgeries through a transoral approach with wearing a divided splint. Since accurate synchronization of the acquired CT data was also achieved for the mandibular body in our case, cutting of mandible bone based on the pre-operative design was successfully and precisely performed under intraoperative navigation guidance. Since the surgical simulation plans could be transferred to the actual surgery intraoperatively with the guidance of real-time navigation, a surgical template with bone cutting guides was not needed in this case.

A 3-D formable titanium mesh and exclusive forming-hand press machine were used to fabricate a patientspecific titanium mesh tray. This method has major advantages over the conventional bending technique with respect to the mechanical strength of the titanium mesh tray, thus preventing titanium mesh fracture. To avoid this serious complication, Yamada et al. [11] reported that combination repair with a titanium reconstruction plate or fabrication of a reinforced titanium mesh tray (e.g. double layered titanium mesh) was necessary for the cases of long-span defect of the mandible. Instead of their materials, a 3-D formable titanium mesh may play critical roles in mandibular reconstruction. Furthermore, mandibular reconstruction using CAD/CAM-mediated trays has advantages of being accurately reproduced and less time-consuming [9]. Therefore, computer-assisted mandibular reconstruction with a CAD/CAM-mediated, patient-specific reconstruction titanium mesh tray becomes a promising method for mandibular reconstruction.

In this case, to achieve satisfactory oral functions, including masticatory performance, mouth opening, and speech, placement of dental implants and prosthodontic rehabilitation are planned as the next step. These sequential treatments are needed for the patient to recover his quality of life, both physiologically and psychologically.

Taken together, integration of preoperative software planning, 3-D surgical simulation combined with intraoperative navigation, and CAD/CAM-mediated patient-specific reconstruction materials improves the surgeons' ability to tailor mandibular reconstruction to individual patients. In the near future, computer-assisted surgery will become standard in craniomaxillofacial surgery.

\section{Acknowledgements}

No grants were received in relation to this paper.

\section{Competing Interests}

The authors declare that they have no competing interests.

\section{References}

[1] Liu, Y.F., Xu, L.W., Zhu, H.Y. and Liu, S.S. (2014) Technical Procedures for Template-Guided Surgery for Mandibular Reconstruction Based on Digital Design and Manufacturing. Biomed Eng Online, 13, 63.

[2] Willems, P.W., van der Sprenkel, J.W., Tulleken, C.A., Viergever, M.A. and Taphoorn, M.J. (2006) Neuronavigation and Surgery of Intracerebral Tumours. Journal of Neurology, 253, 1123-1136. http://dx.doi.org/10.1007/s00415-006-0158-3

[3] Satcher Jr., R.L. (2013) How Intraoperative Navigation Is Changing Musculoskeletal Tumor Surgery. Orthopedic Clinics of North America, 44, 645-656. http://dx.doi.org/10.1016/j.ocl.2013.07.001

[4] Fuller, S.C. and Strong, E.B. (2007) Computer Applications in Facial Plastic and Reconstructive Surgery. Current Opinion in Otolaryngology \& Head \& Neck Surgery, 15, 233-237. http://dx.doi.org/10.1097/MOO.0b013e3281df2c5f

[5] Eiland, M., Habermann, C.R. and Schmelzle, R. (2004) Indications and Limitations of Intraoperative Navigation in 
Maxillofacial Surgery. Journal of Oral and Maxillofacial Surgery, 62, 1059-1063.

[6] Ayoub, N., Ghassemi, A., Rana, M., Gerressen, M., Riediger, D., Hölzle, F. and Modabber, A. (2014) Evaluation of Computer-Assisted Mandibular Reconstruction with Vascularized Iliac Crest Bone Graft Compared to Conventional Surgery: A Randomized Prospective Clinical Trial. Trials, 15, 114. http://dx.doi.org/10.1186/1745-6215-15-114

[7] Liu, Y.F., Xu, L.W., Zhu, H.Y. and Liu, S.S. (2014) Technical Procedures for Template-Guided Surgery for Mandibular Reconstruction Based on Digital Design and Manufacturing. Biomed Eng Online, 13, 63. http://dx.doi.org/10.1186/1475-925X-13-63

[8] Bell, R.B., Weimer, K.A., Dierks, E.J., Buehler, M. and Lubek, J.E. (2011) Computer Planning and Intraoperative Navigation for Palatomaxillary and Mandibular Reconstruction with Fibular Free Flaps. Journal of Oral and Maxillofacial Surgery, 69, 724-732. http://dx.doi.org/10.1016/j.joms.2009.12.040

[9] Wilde, F., Cornelius, C.P. and Schramm, A. (2014) Computer-Assisted Mandibular Reconstruction Using a PatientSpecific Reconstruction Plate Fabricated with Computer-Aided Design and Manufacturing Techniques. Cranial Maxillofac Trauma Reconstruction, 7, 158-166. http://dx.doi.org/10.1055/s-0034-1371356

[10] Ferreira, J.J., Zagalo, C.M., Oliveira, M.L., Correia, A.M. and Reis, A.R. (2014) Mandible Reconstruction: History, State of the Art and Persistent Problems. Prosthetics \& Orthotics International, Published Online.

[11] Yamada, H., Nakaoka, K., Horiuchi, T., Kumagai, K., Ikawa, T., Shigeta, Y., Imamura, E., Iino, M., Ogawa, T. and Hamada, Y. (2014) Mandibular Reconstruction Using Custom-Made Titanium Mesh Tray and Particulate Cancellous Bone and Marrow Harvested from Bilateral Posterior Ilia. Journal of Plastic Surgery and Hand Surgery, 48, 183-190. http://dx.doi.org/10.3109/2000656X.2013.848809

[12] Oh, S.M., Lee, C.Y., Kim, J.W., Jang, C.S., Kim, J.Y. and Yang, B.E. (2013) Condylar Repositioning in Bilateral Sagittal Split Ramus Osteotomy with Centric Relation Bite. Journal of Craniofacial Surgery, 24, 1535-1538. http://dx.doi.org/10.1097/SCS.0b013e31829028be

[13] Ohba, S., Yoshimura, H., Ishimaru, K., Awara, K. and Sano, K. (2014) Application of a Real-Time Three-Demensional Navigation System to Various Oral and Maxillofacial Surgical Procedures. Odontology, Published Online. 